\title{
Stabilization of Induction Motor Drives With Poorly Damped Input Filters
}

\author{
Henrik Mosskull, Johann Galić, and Bo Wahlberg, Fellow, IEEE
}

\begin{abstract}
Efficient torque control of induction motor drives in combination with resonant dc-link input filters can lead to a type of stability problem that is known as negative impedance instability. An often-proposed solution to this problem is the nonlinear system stabilizing controller (NSSC). Stability is usually analyzed under the simplifying assumption of perfect torque control. This indicates that the NSSC stabilizes the drive at any operating point. In this paper, however, we show power laboratory experiments where the NSSC stabilization fails. An improved framework for stability analysis and synthesis of stabilization, based on a linear feedback model of the drive, is therefore proposed. With this approach, effects of time delays can easily be included, and stability margins can be directly established from measurements. To solve the indicated problems with NSSC, a stabilization controller that considers the practical limitations of torque control is derived. In the design of the stabilization controller, the tradeoff between damping and acceptable torque control is also explicitly taken into account. The proposed stabilization scheme is implemented and evaluated on a hardware-in-the-loop simulator as well as in a power laboratory. The results show that the proposed method outperforms the NSSC method.
\end{abstract}

Index Terms-Control design, induction motor drives, stability, torque control.

\section{INTRODUCTION}

$\mathbf{E}$ VEN though induction motors have been around for more than a hundred years, control of these motors is still an active field of research. Some recent publications are, for example, [1]-[3]. The aim of the research is often robust highperformance control of the motor torque, without consideration of the power supply. However, for an induction motor drive with a poorly damped input filter, efficient torque control may lead to oscillating dc-link quantities (see, e.g., [4]-[12]). Such problems are sometimes referred to as negative impedance instabilities and reflect that efficient torque control (more specifically, efficient disturbance rejection) gives the inverter of the drive, as seen from the dc-link, a negative resistance behavior. Negative impedance instabilities are also known from dc-dc converter applications (see, e.g., the recent publications [13] and [14]). With dc-dc converters, the stability problem is often

Manuscript received January 4, 2006; revised January 22, 2007. This work was supported by the Swedish Research Council.

H. Mosskull and J. Galić are with Bombardier Transportation, 72173 Västerås, Sweden (e-mail: henrik.mosskull@se.transport.bombardier.com; johann.galic@se.transport.bombardier.com).

B. Wahlberg is with Automatic Control, Royal Institute of Technology (KTH), 10044 Stockholm, Sweden (e-mail: bo.wahlberg@ee.kth.se).

Color versions of one or more of the figures in this paper are available online at http://ieeexplore.iee.org.

Digital Object Identifier 10.1109/TIE.2007.899912 solved by adding resistance to the input filter. For high-power applications such as induction motor drives that are used for traction, this approach is not feasible due to the large additional power losses. An alternative solution then is to modify the input impedance of the inverter. This means that the power flow of the drive is adapted to variations in the dc-link voltage (i.e., modified disturbance rejection). In [7] and [8], an explicit stabilization scheme is presented, which in [9] is called the nonlinear system stabilizing controller (NSSC). Under the oftenused assumption of perfect torque control (disturbance rejection as well as reference tracking), it can be shown that the NSSC stabilizes the drive at all operating points. However, we will present power laboratory experiments where stabilization with NSSC fails. These results call for improved stability analysis as well as improved stabilization design. For this purpose, a framework for stability analysis based on a linear feedback model of the drive is introduced. This route is also followed in [12], but here, we also consider the torque dynamics to explicitly handle performance requirements. A major advantage with the feedback approach is that stability margins can be accurately estimated directly from measurements. Furthermore, for improved stabilization synthesis, simple dynamic models of the drive, which predict the stability problems, are derived and validated. These models are used to derive a stabilization controller, where also the tradeoff between control performance and damping of the drive is explicitly addressed (cf., [11]). Although the analysis framework can be applied to any torque control strategy, the explicit stabilization algorithm that is presented in this paper is derived to be used with the method indirect self control (ISC) [15], [16]. The results can, however, be extended to other control methods, such as the classical fieldoriented control (FOC, see [17] and [18]).

After a brief introduction to induction motor drives in Section II, the results that are obtained with the assumption of perfect control are reviewed in Section III. Power laboratory experiments showing stability problems with the NSSC are presented in Section IV, and a feedback model for stability analysis and stabilization design is derived in Section V. Stability analysis is performed in Section VI and is followed by the design of stabilization in Section VII. This paper is finally concluded in Section VIII.

\section{INDUCTION MOTOR DRIVE}

An induction motor drive with de supply is depicted in Fig. 1. The three-phase ac stator voltage for the induction motor(s) (one may run several motors in parallel) is generated from $d c$-link voltage $U_{d}(t)$ through a voltage-source inverter (VSI). 


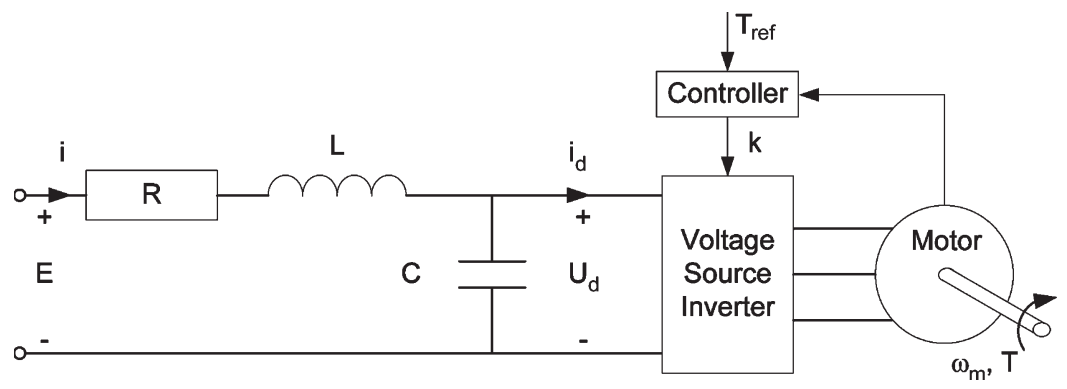

Fig. 1. Induction motor drive.

The dc-link voltage is in turn fed from supply voltage $E(t)$ via an input filter, which is composed of a capacitor with capacitance $C$, together with an inductor with inductance $L$ and resistance $R$. The capacitor is an energy reservoir for the VSI, whereas the inductor is needed to give appropriate suppression of the high-frequency harmonics that are generated by the inverter.

In traction applications, space and weight constraints tend to keep capacitance $C$ of the input filter relatively small. Moreover, to meet regulations on harmonics suppression, or to assure a certain required input impedance of the drive, inductance $L$ has to be relatively large. In combination with a small resistance $R$ to keep the power losses acceptable, the resulting input filters get poorly damped with high resonance peaks at the frequency $\omega_{0}=1 / \sqrt{ }(L C)$. The damping factor of the filter is given by $\zeta=R / 2 \sqrt{ }(C / L)$.

\section{Stabilization With Perfect Control}

In the analysis of induction motor drives, it is often assumed that efficient torque control keeps the motor power unaffected by variations in the dc-link voltage, i.e., product $P(t)=U_{d}(t) i_{d}(t)$ is assumed to be constant (see, e.g., [4]-[6] and [8]-[12]). This assumption means that the expression for the power can be linearized to give

$$
i_{d}(t)=-\frac{i_{d 0}}{U_{d 0}} U_{d}(t)=\underbrace{-\frac{P_{0}}{U_{d 0}^{2}}}_{Y} U_{d}(t)
$$

where subscript " 0 " denotes steady-state values (note that the signals in (1), as in all linearized expressions in this paper, represent deviations from an operating point). Under the assumption of constant power operation, (1) hence forms a linear model of the inverter in Fig. 1 as seen from the dc link, where $Y$ is the input admittance of the inverter. By defining the corresponding equivalent inverter resistance $R_{\text {inv }}=1 / Y$, the drive in Fig. 1 may be modeled by the simple electric circuit in Fig. 2.

From (1), we see that the equivalent resistance is negative at positive power. This follows as, for example, a decrease of dclink voltage must result in an increase of dc-link current to keep the product of dc-link voltage and dc-link current constant. A negative resistance (input admittance) is bad for stability. This

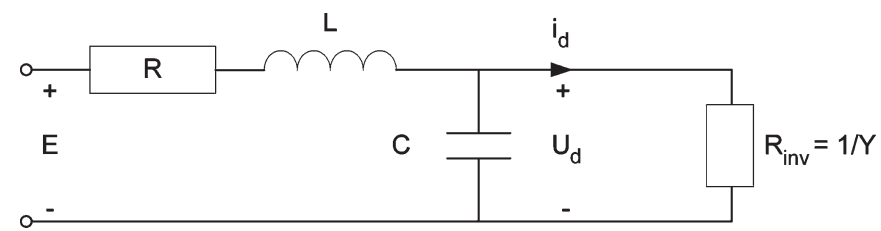

Fig. 2. Linear model of the induction motor drive under the assumption of perfect disturbance rejection (constant power operation). Here, the VSI is modeled by an equivalent resistance $R_{\mathrm{inv}}=1 / Y$.

can, for example, be established from damping factor $\zeta_{\text {drive }}$ of the circuit in Fig. 2, i.e., (cf., [4])

$$
\zeta_{\text {drive }}=(1+Y R)^{-\frac{1}{2}}\left(\zeta+\frac{1}{2} Y \sqrt{\frac{L}{C}}\right) \approx \zeta+\frac{1}{2} Y \sqrt{\frac{L}{C}}
$$

where $\zeta=R / 2 \sqrt{ }(C / L)$ is the damping factor of the input filter alone. From the definition of the admittance in (1), it follows that $Y$ is proportional to $-P_{0}$. The expression for $\zeta_{\text {drive }}$ in (2) then implies that damping decreases with positive power, whereas negative power increases the damping. For the drive to be stable, damping factor $\zeta_{\text {drive }}$ must be positive, which, through (1) and (2), puts the following restriction on the power:

$$
P_{0} \leq \frac{R C}{L} U_{d 0}^{2}
$$

This expression has been used to predict stability in [4]-[6], [8], and [9]. For a drive with data according to the Appendix, the stability condition (3) is only satisfied for powers less than $7 \%$ of the nominal power. Hence, these kinds of stability problems need to be considered for practical operation of the drive.

An intuitive approach to stabilizing the drive is to improve damping by making the inverter instead act like a positive resistance. This means that a decrease in dc-link voltage should result also in a decrease of dc-link current and not an increase, as with the constant power operation. To obtain this, the following modification of the torque reference is suggested to stabilize the drive in [7] and [8] (actually, braking is not considered in [8]):

$$
T_{\mathrm{ref}}(t)= \begin{cases}\left(\frac{U_{d}(t)}{U_{d 0}}\right)^{\rho} T_{\mathrm{ref}}^{\prime}(t), & T_{0} \omega_{m 0} \geq 0 \\ \left(\frac{U_{d 0}}{U_{d}(t)}\right)^{\rho} T_{\mathrm{ref}}^{\prime}(t), & T_{0} \omega_{m 0}<0\end{cases}
$$

where $T_{\text {ref }}^{\prime}$ is an external torque reference, and $\rho \geq 1$ is a design parameter. This approach is called the NSSC in [9]. Note, 


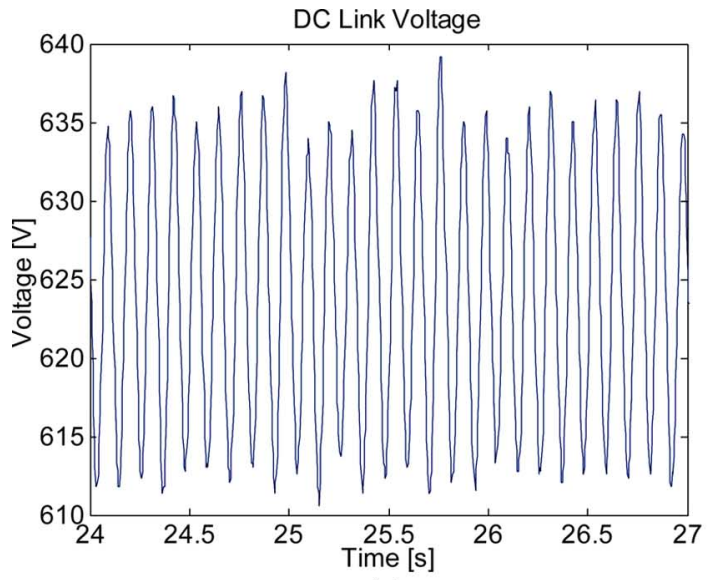

(a)

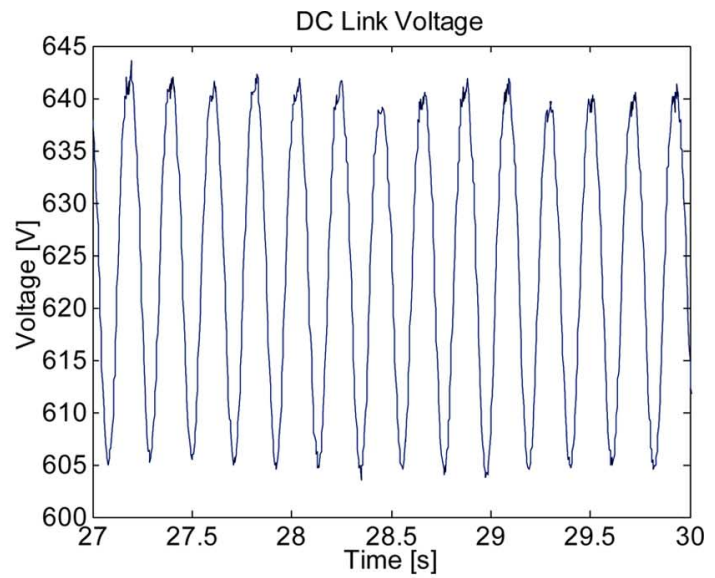

(b)

Fig. 3. Power laboratory registrations of the dc-link voltage of an induction motor drive at (a) 0.7 p.u. speed (817-Hz switching frequency) and (b) 1 p.u. speed (62-Hz switching frequency). The experiments are performed at zero torque with NSSC.

however, that the modified behavior of the inverter means that power is no longer unaffected by disturbances in the dc-link voltage. This will also affect the torque dynamics (see further Section V).

To analytically evaluate the effect of the NSSC, we use the following power balance equation:

$$
i_{d}(t) U_{d}(t)=T(t) \omega_{m}(t)
$$

which is valid if power losses of the inverter and motor are neglected. Apart from perfect disturbance rejection, we also assume perfect reference tracking, i.e., $T(t)=T_{\text {ref }}(t)$, and substitute the stabilization expression (4) in the power balance (5). The relation between the dc-link current and dc-link voltage can then be linearized and the inverter can be modeled by (cf., Fig. 2)

$$
Y= \begin{cases}\frac{(\rho-1) P_{0}}{U_{d 0}^{2}}, & P_{0} \geq 0 \\ \frac{(\rho+1)\left|P_{0}\right|}{U_{d 0}^{2}}, & P_{0}<0 .\end{cases}
$$

Now, $Y \geq 0$ at all powers, and the drive has been stabilized [cf., (2)]. The expression for $Y$ for positive power in (6) is derived also in [8] and [9], and is used to verify the stabilizing effect of the NSSC.

An alternative stabilization method to (4) is proposed in [10], where the torque reference is modified as

$$
T_{\mathrm{ref}}(t)=T_{\mathrm{ref}}^{\prime}(t)+\underbrace{k \frac{T_{0}}{U_{d 0}}}_{K} \underbrace{\frac{p T_{C}}{p T_{C}+1}}_{B} U_{d}(t) .
$$

Here, $k \geq 1$ and $T_{C}$ are constants, and $p$ is the differential operator. With the definitions that are introduced in (7), we can write the stabilization algorithm in the general form

$$
T_{\text {ref }}(t)=T_{\text {ref }}^{\prime}(t)+K(p) B(p) U_{d}(t)
$$

where $K(p)$ is a stabilization controller (in general dynamic), and $B(p)$ is a filter. Filter $B(p)$ is needed to avoid constant offsets in the dc-link voltage from giving constant torque contributions, i.e., the low-frequency components of the stabilization should be removed. It may also be advantageous to avoid exiting the torque reference with too high frequencies due to problems with limited control bandwidth (or to avoid aliasing in a digital implementation). This suggests the use of a bandpass filter, instead of the high-pass filter that is proposed in (7). Note that such a bandpass filter is also needed with (4), although not explicitly included in the expression.

To show the similarity between the stabilization schemes that are given by (4) and (7), we linearize the expression for the torque reference (4). It then follows that (4) can be written as (8) with [cf., (7)]

$$
K=\frac{\rho\left|T_{0}\right| \operatorname{sgn}\left(\omega_{m 0}\right)}{U_{d 0}} .
$$

The scheme in (7) is only proposed at positive power, which means that, in a linear sense, (7) is equivalent to NSSC with $k=\rho$. The stabilizing effect of (7) is established in [10] under the assumption of perfect reference tracking.

1) Remark: In [4], an expression that is similar to (8) is proposed for the $q$-component of the stator current. However, no explicit expression for the stabilization controller is given.

\section{UNSTABLE EXAMPLES}

The simplified analysis in the previous section showed that stabilization with NSSC would give a stable drive at all operating points. This result is, however, contradicted by Fig. 3, showing power laboratory measurements of the dc-link voltage of an induction motor drive using NSSC. The experiments were performed at zero torque and two different speeds in a power laboratory at Bombardier Transportation, Västerås, Sweden. Here, the VSI feeds four induction motors in parallel. See the Appendix for further data of the drive.

In [4]-[6], [8], and [9], condition (3) is used to state that stabilization is not needed at zero torque (note, e.g., that the NSSC does not affect the system at zero torque). However, this result is derived under the assumption of constant power operation, i.e., perfect disturbance rejection. This never perfectly holds in practice, which is the explanation for the instabilities that are seen in Fig. 3. In order to further analyze these stability 


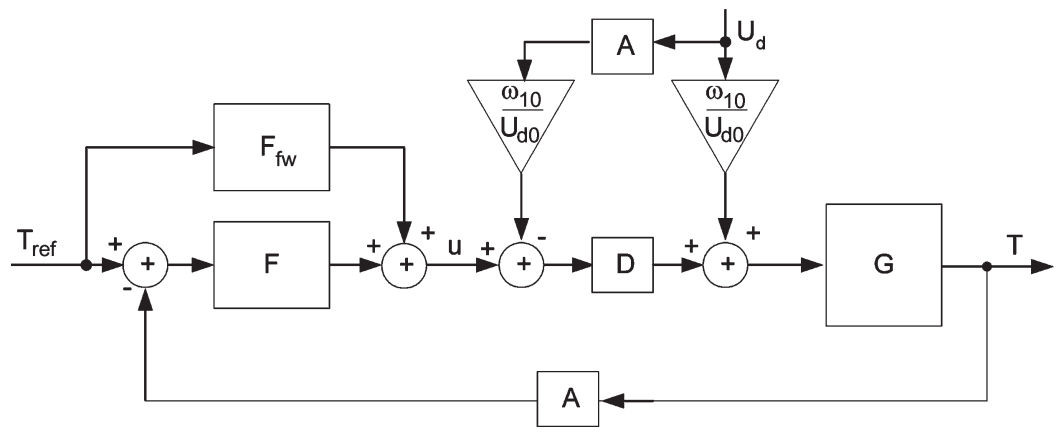

Fig. 4. Block diagram of the induction motor controlled with ISC.

problems, we introduce a different framework, where the drive is represented as a feedback system. This approach implies not only that time delays can easily be included but also that the models that are needed for analysis can be directly identified from measurements, thus assuring accurate (linear) analysis.

In [17], an example where NSSC fails at large torque is also given. Here, the problem is imperfect reference tracking due to limited control bandwidth.

\section{Linear FeEdBACK Model of The DRIVE}

In this section, we derive a linear feedback model of the drive, where we relax the assumption of perfect control. In contrast to the analysis in Section III, this treatment requires details of the underlying torque control. We will also show that the approach can be used with models that are identified from measurements, thus giving very accurate stability analysis.

We start the model derivation by describing the torque as

$$
T(t)=G_{c}(p) T_{\mathrm{ref}}(t)+G_{d}(p) U_{d}(t)
$$

where $G_{c}(p)$ and $G_{d}(p)$ are linear transfer functions (the speed is considered constant and does therefore not influence the torque). In (10), we hence assume neither perfect disturbance rejection (constant power operation), as in [4]-[6] and [8]-[12], nor perfect reference tracking, as done in [8]-[10].

In practice, limited torque control bandwidth implies that operation with near-constant power is not accomplished through feedback control alone. The inverter switching commands are therefore often directly modified by normalizing the inverter modulation index by a measured dc-link voltage. That means that we will consider the stator voltage space vector to be given by (neglecting harmonics)

$$
\boldsymbol{u}_{s}(t) \approx \frac{\boldsymbol{u}_{\mathrm{sref}}\left(t-T_{d}\right)}{\bar{U}_{d}\left(t-T_{d}\right)} U_{d}(t)
$$

where $T_{d}$ is the time delay due to pulsewidth modulation (PWM), and an average dc-link voltage is used to suppress switching frequency harmonics. Using (11) and the control method ISC, the model for the torque control in Fig. 4 is derived in [17]. Note that the feedforward compensation of dc-link voltage disturbances in Fig. 4 corresponds to a linearization of (11).
The block $G$ in Fig. 4 represents the induction motor, whereas the blocks $F$ and $F_{\mathrm{fw}}$ model the ISC control. The Laplace transfer functions $F_{\mathrm{fw}}, F$, and $G$ are given by

$$
\begin{aligned}
F_{\mathrm{fw}} & =\frac{2}{3} \frac{R_{r}}{n_{p} \psi_{r 0}^{2}} \\
F(s) & =\frac{2}{3} \frac{R_{r}}{n_{p} \psi_{r 0}^{2}}\left(K_{p}+\frac{1}{s} K_{i}\right) \\
G(s) & =\frac{3}{2} \frac{n_{p} \psi_{r 0}^{2}}{R_{r}\left(s T_{\sigma}+1\right)}
\end{aligned}
$$

where $n_{p}$ is the number of pole pairs of the induction motor, $\psi_{r 0}$ is the steady-state rotor flux magnitude, $R_{r}$ is the rotor resistance, and $T_{\sigma}=L_{\sigma} / R_{r}$ is the rotor leakage time constant. Finally, $D$ and $A$ in Fig. 4 denote a time delay due to PWM and an average over a pulse period, respectively, and $\omega_{10}$ is the operating point stator frequency.

From Fig. 4, it follows that the transfer functions $G_{c}$ and $G_{d}$ in the torque (10) can be written as

$$
\begin{aligned}
G_{c} & =\frac{G D\left(F_{\mathrm{fw}}+F\right)}{1+G D F A} \\
G_{d} & =\frac{\omega_{10}}{U_{d 0}} \frac{G(1-D A)}{1+G D F A} .
\end{aligned}
$$

In order to relate the ac and dc sides of the inverter in Fig. 1, we use the power balance (5). If we consider the motor speed $\omega_{m}$ to be fixed, this equation can be linearized to give

$$
i_{d}(t)=\frac{\omega_{m 0}}{U_{d 0}} T(t)-\frac{P_{0}}{U_{d 0}^{2}} U_{d}(t)
$$

where we used $P_{0}=T_{0} \omega_{m 0}$. It then follows from (8), (10), and (14) that

$i_{d}(t)=\frac{\omega_{m 0}}{U_{d 0}} G_{c} T_{\mathrm{ref}}^{\prime}(t)+\underbrace{(\frac{\omega_{m 0}}{U_{d 0}} \overbrace{\left(G_{d}+B K G_{c}\right)}^{\hat{G}_{d}(p)}-\frac{P_{0}}{U_{d 0}^{2}})}_{Y(p)} U_{d}(t)$

where we again defined the inverter input admittance $Y(p)$ as the linear mapping between the dc-link voltage and the dc-link current. Note that, without stabilization, this expression reduces to the static expression $Y=-P_{0} / U_{d 0}^{2}$ in (1) under the 


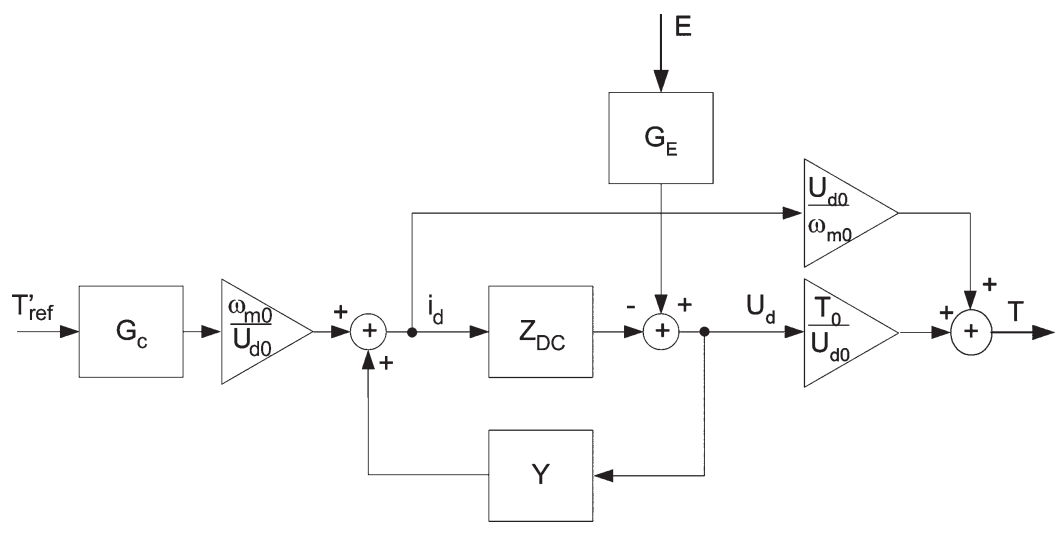

Fig. 5. Linear feedback model of an induction motor drive.

assumption of perfect disturbance rejection, i.e., $G_{d}=0$. From (15), we also see that the stabilization modifies the disturbance transfer function (response from $U_{d}$ to $T$ ) from $G_{d}$ to $\hat{G}_{d}=$ $G_{d}+B K G_{c}$.

The dc-link voltage and the dc-link current are also related through the input filter in Fig. 1. This implies that the dc-link voltage depends on the line voltage and the dc-link current as

$$
U_{d}(t)=G_{E}(p) E(t)-Z_{\mathrm{dc}}(p) i_{d}(t)
$$

where the transfer functions $G_{E}(s)$ and $Z_{\mathrm{dc}}(s)$ are given by

$$
\begin{aligned}
G_{E}(s) & =\frac{1}{s^{2} C L+s C R+1} \\
Z_{\mathrm{dc}}(s) & =\frac{s L+R}{s^{2} C L+s C R+1} .
\end{aligned}
$$

Equations (10), (14), and (16) can now be combined to give the linear feedback model of the drive in Fig. 5. The feedback loop of the model consists of input filter impedance $Z_{\mathrm{dc}}$ and inverter input admittance $Y$. Hence, by including the coupling between the dc-link voltage and the torque, Fig. 5 implies that the torque equation can be written as in

$$
\begin{aligned}
T(t)=\left(1-\frac{Y+\frac{P_{0}}{U_{d 0}^{2}}}{1+Y Z_{\mathrm{dc}}} Z_{\mathrm{dc}}\right) & G_{c} T_{\mathrm{ref}}^{\prime}(t) \\
& +\frac{U_{d 0}}{\omega_{m 0}} \frac{Y+\frac{P_{0}}{U_{d 0}^{2}}}{1+Y Z_{\mathrm{dc}}} G_{E} E(t) .
\end{aligned}
$$

We assume that the internal torque control is stable, i.e., that $G_{c}$ and $G_{d}$ are stable transfer functions, which, through (15), means that $Y$ is also stable. Then, all the individual blocks in Fig. 5 are stable, and we can use the simplified Nyquist stability criterion to examine the stability of the drive, i.e., the drive is stable if the Nyquist curve of the loop gain $Y Z_{\mathrm{dc}}$ does not encircle point -1 in the complex plane. From a robustness point of view, it is advantageous to use a feedback model and the Nyquist stability criterion compared to the eigenvalue analysis that is performed in, e.g., [5], as, for example, time delays are easy to include. Similar approaches to stability analysis are taken for dc-dc converters in, e.g., [13] and for traction power supply grids in [19].
For accurate stability analysis, we may directly estimate the inverter input admittance $Y$ in Fig. 5 from measurements (see also [20] and [21]). Even though the admittance in principle can be identified from a real setup in a power laboratory, we will here use "measurements" that are generated from a hardware-in-the-loop simulator to determine $Y$. This means that real control hardware and software are used, whereas the inverter and induction motors are simulated in "real time" (sampling interval of $60 \mu \mathrm{s}$ ). The inverter and induction motors are relatively well defined by mathematical models, and the simulation results show good agreement with power laboratory measurements. These kinds of simulations for traction applications have prior been presented in, for example, [22] and allow for much faster and more flexible experiments compared to using a real drive.

\section{Stability ANALYSis}

Due to the resonant character of input filter impedance $Z_{\mathrm{dc}}$, a negative inverter input admittance (as considered in Section III) generates a loop gain $Y Z_{\mathrm{dc}}$ that may encircle point -1 in the complex plane. With a more general transfer function model of $Y$, a nonpassive admittance may be enough to cause instability. In particular, the values of the admittance at the resonance frequency of the input filter, which is here denoted as $\omega_{0}$, are crucial. According to (15), positive power pushes the admittance into the left half-plane, hence making the admittance "less passive" and consequently reducing stability margins. However, even at zero torque, the admittance may in fact be nonpassive, as seen by the Nyquist curves of the admittance in Fig. 6 . Here, the Nyquist curves that are obtained from hardwarein-the-loop simulations are shown in the left figure, and the corresponding Nyquist curves that are obtained with the simple model of $Y$ in (15) are shown in the right figure. The admittance has been calculated for several motor speeds, where the bold dashed and solid curves correspond to the operating points that are used in Fig. 3(a) and (b), respectively.

At zero torque, the NSSC offers no stabilization as $K=0$ in (9), and the admittance is therefore proportional to the disturbance transfer function $G_{d}$ [see (15)]. The nonzero $Y$ in Fig. 6 consequently shows that $G_{d} \neq 0$, which is plausible from the expression for $G_{d}$ in (13), as time delays always are present in practice. Even though the disturbance suppression 


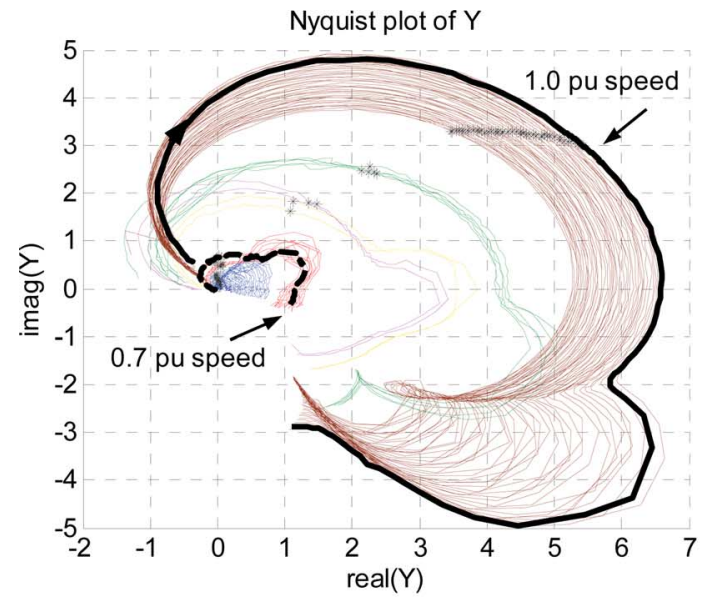

(a)

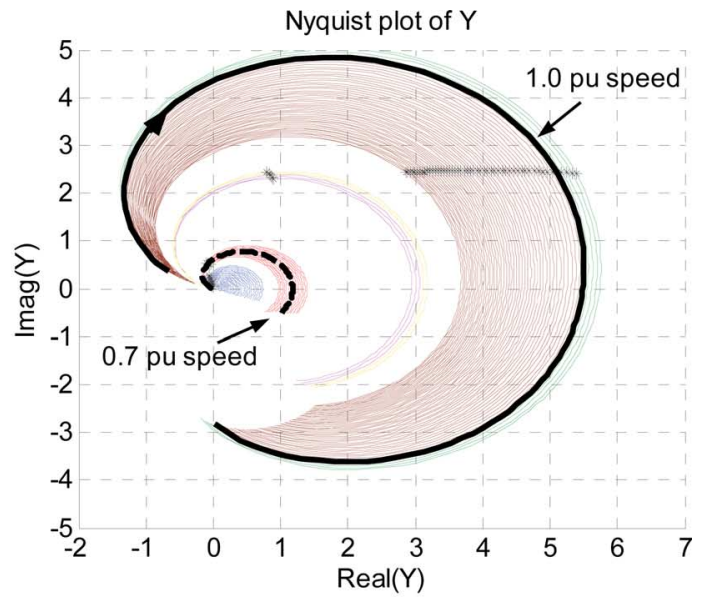

(b)

Fig. 6. Nyquist curves of inverter input admittance $Y$ at zero torque at several motor speeds with (a) identified (hardware-in-the-loop simulator) and (b) simple dynamic models of the inverter input admittance. The bold dashed curves correspond to 0.7 p.u. speed, and the bold solid curves correspond to 1 p.u. speed. The stars correspond to the values at the resonance frequency of the input filter.

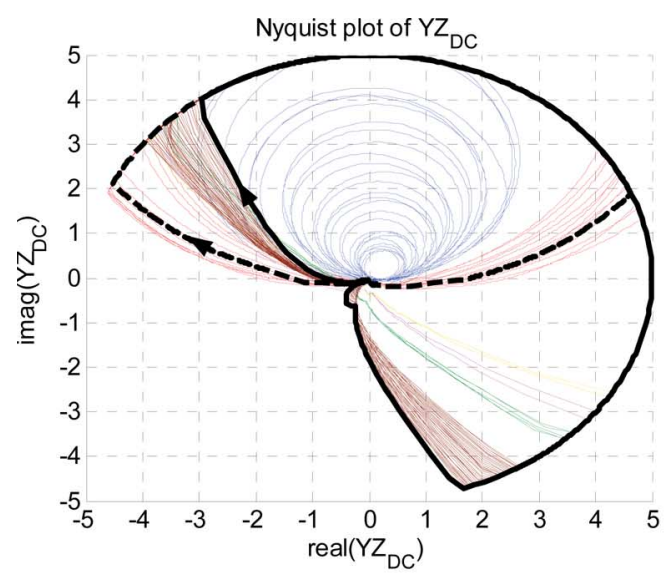

(a)

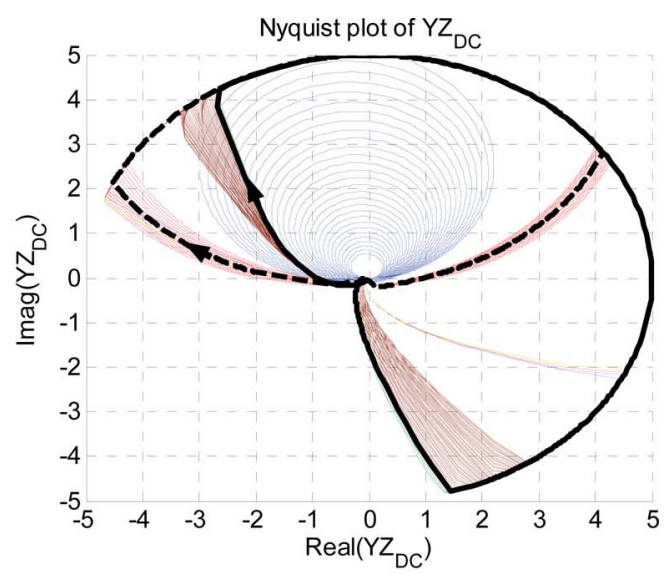

(b)

Fig. 7. Nyquist curves of loop gain $Y Z_{\mathrm{dc}}$ at zero torque at several motor speeds with (a) identified (hardware-in-the-loop simulator) and (b) simple dynamic models of the inverter input admittance. The bold dashed curves correspond to 0.7 p.u. speed, and the bold solid curves correspond to 1 p.u. speed. The bold curves encircle (or passes close to) point -1 , which indicates instability (or very poor stability margin). Note that the magnitudes are limited to five for visibility reasons.

is not perfect, the feedback factor $1-D A$ and the feedback torque control not only reduce the gain of $G_{d}$ but also increase its phase shift. The large phase-shift makes $G_{d}$, and hence $Y$, nonpassive. For the dashed curve, the admittance is not far into the left half-plane, but the admittance is nonpassive at $\omega_{0}$ (values marked with asterisks). As this value of $Y$ is multiplied by the large resonance peak of the input filter, the loop gain $Y Z_{\mathrm{dc}}$ encircles point -1 . This is seen in Fig. 7, where the left figure again shows results that are obtained from hardware-in-the-loop simulations and the figure to the right shows results using the simple dynamic model. For the bold solid curve in Fig. 6, the admittance is actually passive at the resonance frequency, but the curves go rather far into the left half-plane at frequencies that are close to $\omega_{0}$. As seen in Fig. 7, the Nyquist curve of the loop gain $Y Z_{\mathrm{dc}}$ does not encircle point -1 but passes very close to it. This indicates a very poorly damped closed-loop system. We conclude that analysis using the feedback model predicts the instabilities (or very poor damping) that are shown in Fig. 3.
Note that the instabilities at zero torque are not due to specifically using ISC (see, e.g., the similar analysis using FOC in [18]). The problem is instead caused by a nonpassive $Y$ that is close to $\omega_{0}$, due to efficient but not perfect disturbance rejection. We see in Fig. 6 that the admittance is passive at high frequencies as disturbance rejection deteriorates with increasing disturbance frequency. Stability problems at zero torque are therefore more likely with an input filter with a low rather than a high resonance frequency.

For maximum stability margins, inverter input admittance $Y$ should be positive real, at least around the resonance frequency of the input filter. Very loosely speaking, this means that the gain of the modified disturbance transfer function $\hat{G}_{d}$ must exceed $P_{0} / U_{d 0}^{2}$ (for $P_{0}>0$ ) [see (15)]. However, the term $Y+P_{0} / U_{d 0}^{2}$ in (18) is proportional to $\hat{G}_{d}$. A large gain of $\hat{G}_{d}$ therefore also makes the torque dynamics deviate from the desired transfer function $G_{c}$. For this reason, the gain of $\hat{G}_{d}$ should be chosen to be as small as possible while still making the inverter input admittance positive real (or at least 
guaranteeing satisfying stability margins). We will assure a small gain of $\hat{G}_{d}$ by minimizing the gain of stabilization controller $K$. Note also that removing the feedforward compensation (11) would cancel much of the negative impedance behavior, i.e., improving stability. On the other hand, it gives unacceptable control performance due to a large gain of $G_{d}$.

\section{Stabilization Controller Design}

We now continue with stabilization design to solve the stability problems with NSSC that is presented in Section IV. In this paper, we focus on the torque control method ISC. A similar treatment of stabilization with a classical field-oriented controller can be found in [17] and [18].

From the preceding discussion, we may view stabilization of the drive as appropriately shaping the inverter input admittance via stabilization controller $K$. In order to achieve a desired inverter input admittance $Y_{d}$, it follows from the expression for the admittance in (15) that stabilization controller $K$ should be chosen as

$$
K=G_{c}^{-1} \frac{U_{d 0}}{\omega_{m 0}}\left(Y_{d}+\frac{P_{0}}{U_{d 0}^{2}}\right)-G_{c}^{-1} G_{d} .
$$

Note that we neglect filter $B$ during controller synthesis and hence assume that $B$ is chosen to give small effect around $\omega_{0}$. During controller evaluation, filter $B$ will be considered though (see data for $B$ in the Appendix).

We previously discussed the selection of $Y_{d}$ based on requirements on stability and torque control performance (positive real $Y_{d}$ with the minimum gain of $K$ ). The third requirement that we impose on $Y_{d}$ is that the resulting stabilization controller (19) should be reasonably simple to implement. These three design objectives are partly conflicting, and an acceptable compromise has to be found. In order to do this, the design process is performed in three steps. In the first step, a simple controller that makes the admittance real and positive, i.e., obtaining optimum stability margin, is derived. While keeping the simple expression of the stabilization controller, the stability margin is then slightly reduced in the second step to improve control performance. This controller, however, still requires the inversion of torque transfer function $G_{c}$, which is not exactly possible in practice. In the final step, appropriate approximations of this inversion are therefore discussed.

\section{A. Design for Maximum Stability Margin}

With ISC, we use the expressions for $G_{c}$ and $G_{d}$ that are given by (13) to rewrite the second term of the ideal stabilization controller in (19) as

$$
-G_{c}^{-1} G_{d}=\frac{\omega_{10}}{U_{d 0}}\left(1-G_{c}^{-1}\right) \frac{G(1-D A)}{1-G D F(1-A)-G D F_{\mathrm{fw}}} .
$$

For small pulse periods (small time delays), we can approximate $D \approx 1$ and $1-A \approx 0$ in the denominator of (20). If we further approximate the product $D A$ by an equivalent time delay of $T_{\mathrm{da}}$ seconds, we can also use $1-D A \approx p T_{\mathrm{da}}$ in (20).
This means that we, after some rearrangement, can write

$$
\begin{array}{r}
K=\frac{U_{d 0}}{\omega_{m 0}} G_{c}^{-1}\left[Y_{d}-\left(\frac{3}{2}\left(\frac{\psi_{r 0}^{2}}{U_{d 0}}\right)^{2} \frac{\omega_{r 0} \omega_{10} T_{\mathrm{da}}}{L_{\sigma}}-\frac{P_{0}}{U_{d 0}^{2}}\right)\right] \\
+\frac{3}{2} \frac{n_{p} \psi_{r 0}^{2}}{U_{d 0} L_{\sigma}} \omega_{10} T_{\mathrm{da}}
\end{array}
$$

where $\omega_{r 0}=n_{p} \omega_{m 0}$. To reach a positive real inverter input admittance (for maximum stability margins) with a simple stabilization controller, we propose to choose

$$
Y_{d}=\frac{3}{2}\left(\frac{\psi_{r 0}}{U_{d 0}}\right)^{2} \frac{\omega_{r 0} \omega_{10} T_{\mathrm{da}}}{L_{\sigma}}+ \begin{cases}0, & P_{0}>0 \\ \frac{\left|P_{0}\right|}{U_{d 0}^{2}}, & P_{0} \leq 0\end{cases}
$$

which minimizes the influence of $G_{c}^{-1}$ in the expression of the stabilization controller (21). This is positive from an implementation point of view.

The admittance (22) is positive real, apart from cases where the stator frequency and rotor speed have different signs, which may happen around zero speed. This is not a problem as the magnitude of the admittance is small enough not to cause any problems at those speeds $\left(\left|Y Z_{\mathrm{dc}}\right|<1\right)$. In order to obtain the admittance (22), it follows from (21) that the stabilization controller should be chosen as

$$
K=\frac{3}{2} \frac{n_{p} \psi_{r 0}^{2}}{U_{d 0} L_{\sigma}} \omega_{10} T_{\mathrm{da}}+ \begin{cases}G_{c}^{-1} \frac{T_{0}}{U_{d 0}}, & T_{0} \omega_{m 0}>0 \\ 0, & T_{0} \omega_{m 0} \leq 0\end{cases}
$$

The first term in (23) is needed to compensate for a nonpassive disturbance transfer function $G_{d}$, whereas the second term corresponds to the stabilization controller (9) with the difference that it also considers nonperfect reference tracking (i.e., $G_{c} \neq 1$ ).

\section{B. Improved Control Performance}

We will now reduce the gain of the stabilization controller to improve torque control performance. To keep the simple expression of the stabilization controller, the gain reduction is done by simply multiplying the first term of the expression (23) by a constant factor $K_{0} \leq 1$. The resulting inverter input admittance then becomes

$$
\begin{aligned}
Y=\frac{3}{2}\left(\frac{\psi_{r 0}}{U_{d 0}}\right)^{2} \frac{\omega_{r 0} \omega_{10} T_{\mathrm{da}}}{L_{\sigma}}(1- & \underbrace{\left(1-K_{0}\right) G_{c}}_{\Delta}) \\
& + \begin{cases}0, & P_{0}>0 \\
\frac{\left|P_{0}\right|}{U_{d 0}^{2}}, & P_{0} \leq 0 .\end{cases}
\end{aligned}
$$

Since the admittance (24) is no longer real with $K_{0}<1$, the stability margins are reduced. This is the price that we pay for reducing the gain of the stabilization controller and hence improving control performance.

To derive an appropriate value for $K_{0}$, we, for simplicity, assume that $\Delta$ in (24) has constant magnitude that is independent of the phase (of course, this is not true for analytic functions). At positive power, where stabilization is most important, we note that $\arg Y=\arg (1-\Delta)$. If we demand that the phase 


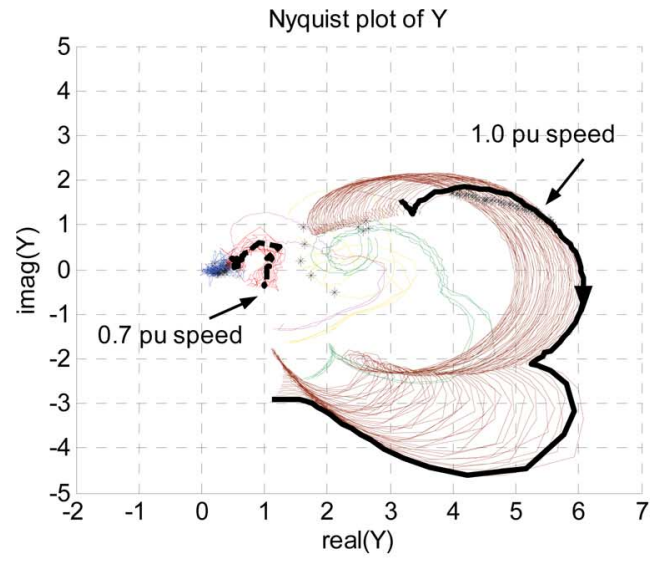

(a)

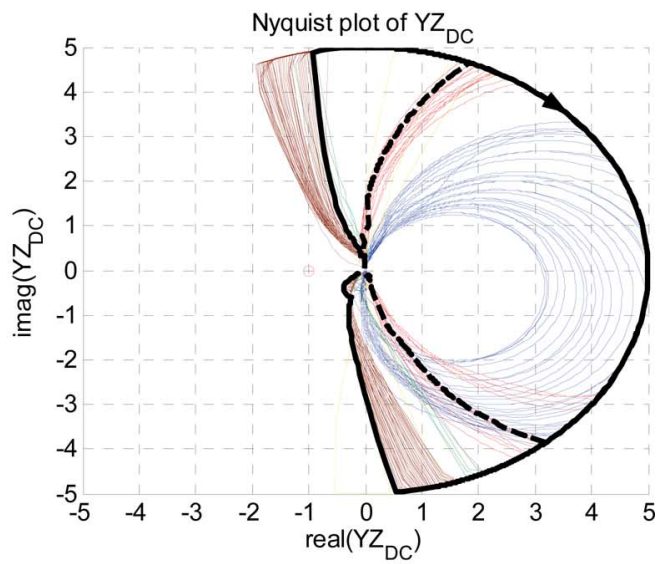

(b)

Fig. 8. Nyquist curves of (a) inverter input admittance $Y$ and (b) loop gain $Y Z_{\mathrm{dc}}$ with stabilization at zero torque and several motor speeds. The admittances are identified using the hardware-in-the-loop simulator. Note that the magnitudes in (b) are limited to five for visibility reasons.

shift of the admittance $Y$ stays less than $\pm 45^{\circ}$, some geometry then gives us that the magnitude of $\Delta$ has to be less than $1 / \sqrt{ } 2$. If we allow a resonance peak of $\sqrt{ } 2$ of $G_{c}$, then the phase requirement implies that $|\Delta| \leq \sqrt{ } 2\left(1-K_{0}\right)<1 / \sqrt{ } 2$. Due to the relatively large allowed resonance peak, we propose to set $K_{0}$ equal to the limit, i.e., $K_{0}=0.5$.

\section{Approximation of $G_{c}^{-1}$}

The first step in the selection of the stabilization controller (23) was taken to give the drive maximum stability margins, i.e., to make the inverter input admittance positive real. However, for positive power, this requires the inversion of $G_{c}$, which is not possible to exactly compute in practice due to, for example, time delays. Therefore, an approximation of the inverse has to be used. If we denote the approximation $F_{\text {lead }}$, the inverter input admittance (24) with $K_{0}=0.5$ is changed to

$$
\begin{aligned}
& Y_{d}=\frac{3}{2}\left(\frac{\psi_{r 0}}{U_{d 0}}\right)^{2} \frac{\omega_{r 0} \omega_{10} T_{\mathrm{da}}}{L_{\sigma}}\left(1-\frac{1}{2} G_{c}\right) \\
&+ \begin{cases}\frac{P_{0}}{U_{d 0}^{2}}\left(G_{c} F_{\text {lead }}-1\right), & P_{0}>0 \\
\frac{\left|P_{0}\right|}{U_{d 0}^{2}}, & P_{0} \leq 0 .\end{cases}
\end{aligned}
$$

In [23], the inverse of $G_{c}$ is simply neglected, i.e., $F_{\text {lead }}=1$. From (25), we see that this means that the second term of the resulting inverter input admittance will contribute with negative real part above the bandwidth of the torque control. However, if the phase shift of transfer function $G_{c}$ at $\omega_{0}$ is moderate, the phase of the inverter input admittance is normally small enough to keep the drive stable (the positive real part of the first term is larger than the negative part of the second term). Neglecting $G_{c}^{-1}$ also decreases the gain of the stabilization controller above the bandwidth of $G_{c}$. As long as stability is preserved, the reduction of stability margins due to the nonreal admittance may hence be desirable for control performance. Although $F_{\text {lead }}=1$ works well in most situations, there are also cases where better approximation of the inverse of $G_{c}$ is needed. One such case is treated in [24], where a lead filter with positive phase shift around the resonance frequency of the input filter is needed in order to stabilize the drive.

\section{Practical Stabilization Controllers}

Summarizing the results from the previous sections, i.e., the expression for the stabilization controller giving maximum stability margins, gain reduction $K_{0}=0.5$, and the approximation of $G_{c}^{-1}$ by filter $F_{\text {lead }}$, we obtain the following final expression for the stabilization controller:

$$
K=\frac{3}{4} \frac{n_{p} \psi_{r 0}^{2}}{U_{d 0} L_{\sigma}} \omega_{10} T_{\mathrm{da}}+ \begin{cases}\frac{T_{0}}{U_{d 0}} F_{\text {lead }}, & P_{0}>0 \\ 0, & P_{0} \leq 0\end{cases}
$$

The stabilization controller (26) makes a good compromise between damping and control performance but is also easy to implement.

\section{E. Results}

The influence of the stabilization controller (26) (with $F_{\text {lead }}=1$ ) is illustrated in Fig. 8. The first figure shows that the inverter input admittance has been shifted into the right halfplane (cf., Fig. 6), and the second figure shows that loop gains $Y Z_{\mathrm{dc}}$ no longer encircle point -1 (cf., Fig. 7). We can hence conclude that the drive has been stabilized. This result is also verified for the speeds that are represented by the dashed and solid bold curves through the power laboratory recordings that are shown in Fig. 9, where the stabilization (26) is turned on in the middle of the figures (cf., Fig. 3). The power laboratory experiments show that the dc-link voltage oscillation (limit cycle) is suppressed by the stabilization in both cases. It can also be shown that the stabilization controller stabilizes the drive in driving and braking as well.

To evaluate the torque control performance, step responses using the proposed stabilization controller and the NSSC are shown in Fig. 10, together with step responses with a constant dc-link voltage. The responses are generated with the hardware-in-the-loop simulator, and it is seen that the proposed 


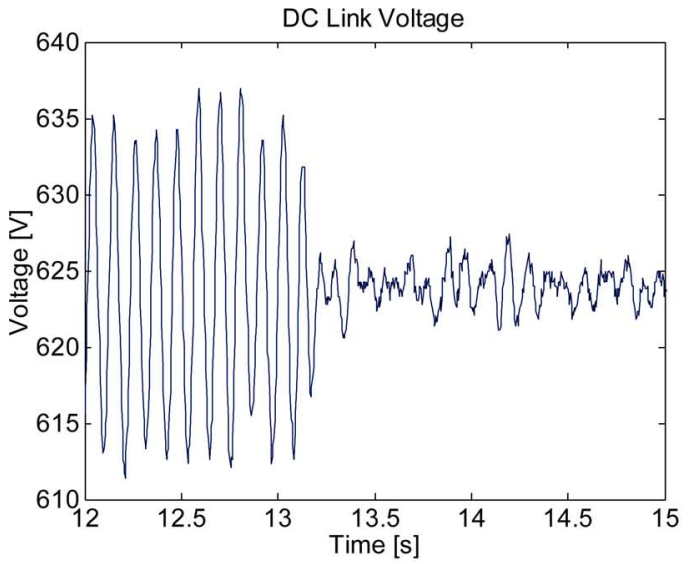

(a)

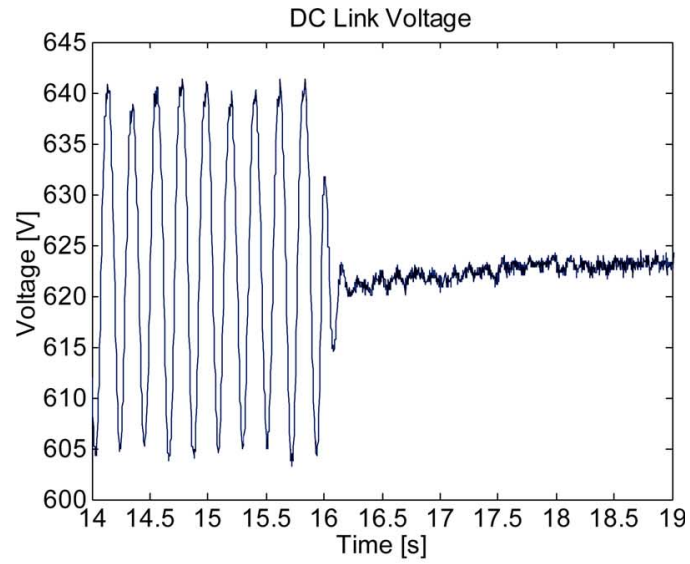

(b)

Fig. 9. Power laboratory registrations showing the dc-link voltage without and with stabilization at (a) 0.7 p.u. speed and (b) 1 p.u. speed with zero torque. Note that the NSSC does not give any stabilization at these operating points.

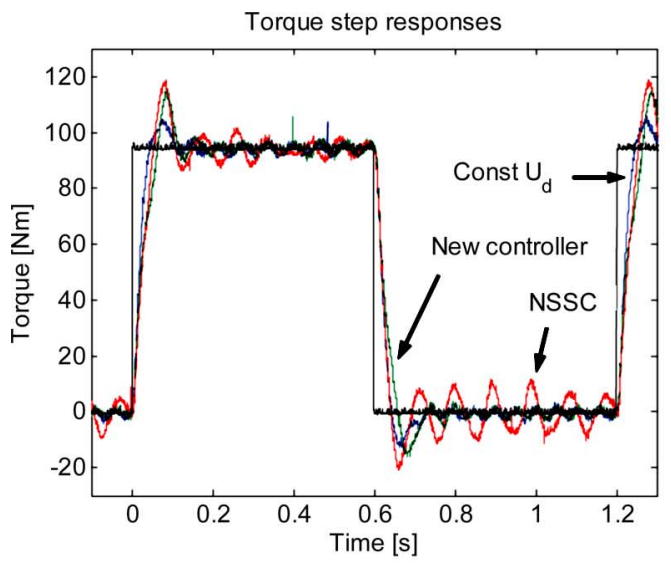

(a)

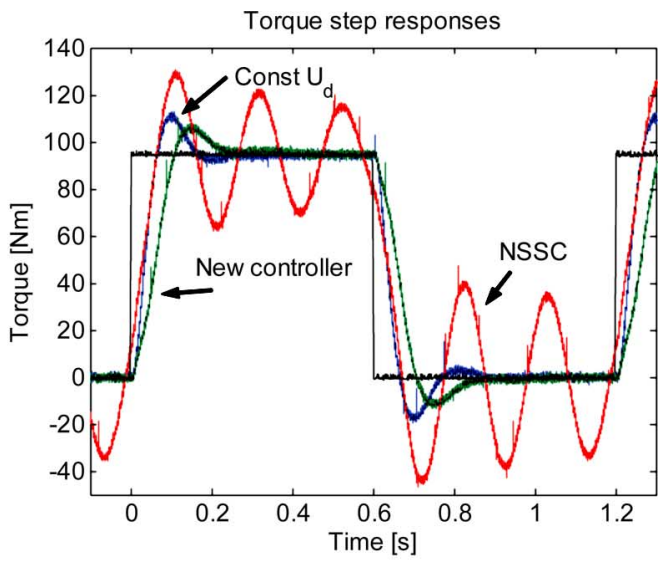

(b)

Fig. 10. Torque step responses generated by the hardware-in-the-loop simulator at (a) 0.7 p.u. speed and (b) 1 p.u. speed. Step responses are shown with the proposed stabilization controller and the NSSC, together with the step responses with constant dc-link voltage.

stabilization controller outperforms the NSSC and gives step responses close to the reference dynamics.

\section{CONCLUSION}

Power laboratory experiments have been presented where the NSSC fails to stabilize an induction motor drive. This implies that the common assumption of perfect torque control (constant power operation) in connection with the stability analysis of induction motor drives is inadequate. An alternative analysis and synthesis framework has therefore been presented, where the drive is represented as a linear feedback system. Here, stability margins may be directly estimated from measurements, thus assuring accurate results.

To solve the presented problem with NSSC, a new stabilization controller was designed, where the nonperfect properties (e.g., due to inevitable time delays) of the torque control were considered. During design, the tradeoff between damping of the system and torque control performance was also taken into account. The designed controller was shown to stabilize the drive and hence outperform the NSSC.

\section{APPENDIX}

All examples in this paper were generated with the following data:

Induction motor ( $\Gamma$-model parameters):

$\begin{array}{ll}\text { Stator resistance } & R_{s}=23.6 \mathrm{~m} \Omega ; \\ \text { Rotor resistance } & R_{r}=16.6 \mathrm{~m} \Omega ; \\ \text { Leakage inductance } & L_{\sigma}=0.94 \mathrm{mH} ; \\ \text { Magnetizing inductance } & L_{m}=7.6 \mathrm{mH} ; \\ \text { Number of pole pairs } & n_{p}=2 ; \\ \text { Nominal speed (electrical) } & \omega_{b}=77.8 \mathrm{~Hz} ; \\ \text { Nominal power } & P_{0}=75 \mathrm{~kW} ; \\ \text { Nominal stator flux } & \Psi_{0}=0.82 \mathrm{Vs} .\end{array}$

Input filter:

$$
\begin{aligned}
& \text { - } R=14 \mathrm{~m} \Omega \\
& \text { - } C=24 \mathrm{mF} \\
& \text { - } L=6 \mathrm{mH} \\
& \text { - } \omega_{0}=83.3 \mathrm{rad} / \mathrm{s} \\
& -\quad \zeta=0.014
\end{aligned}
$$

The switching frequency that was used in the example is varied as a function of stator frequency, as shown in Fig. 11(a). 


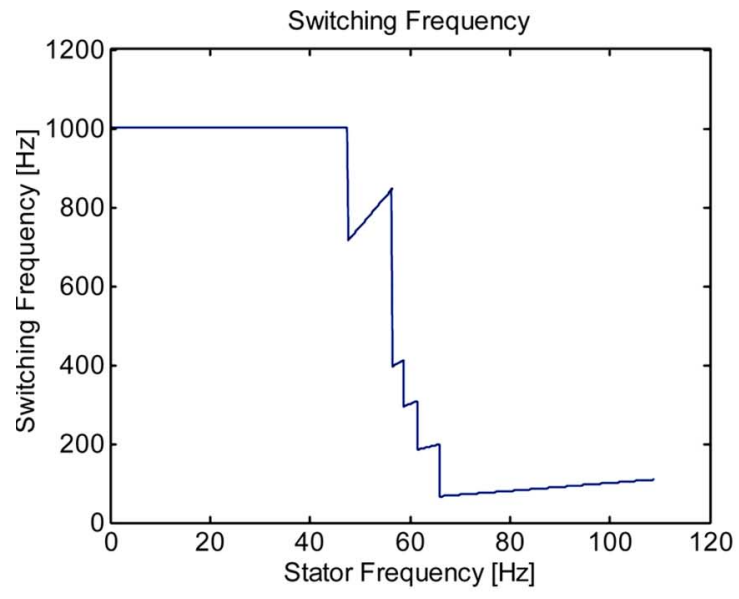

(a)

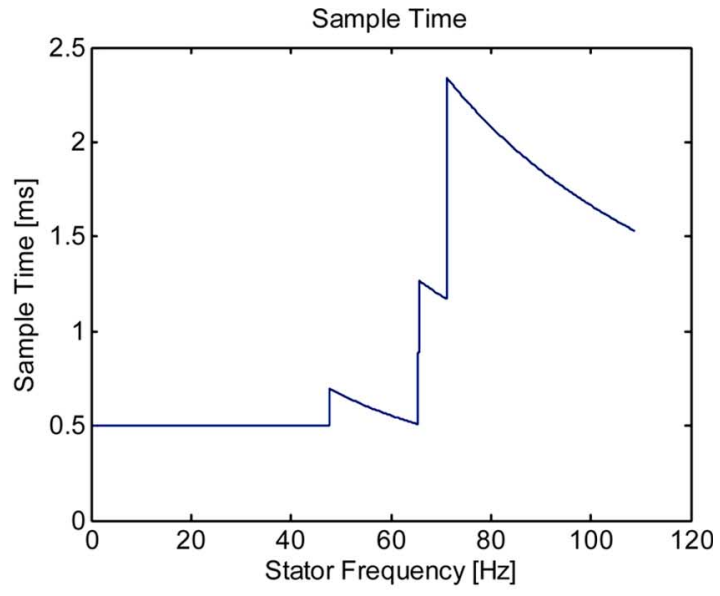

(b)

Fig. 11. (a) Switching frequency and (b) sample time as a function of stator frequency.

Sampling is synchronized to the switching frequency and leads to a sample time variation, as shown in Fig. 11(b). The nominal dc-link voltage was set to $630 \mathrm{~V}$. The cutoff frequencies of bandpass filter $B$ were set to 1 and $80 \mathrm{~Hz}$, respectively.

\section{REFERENCES}

[1] H. Abu-Rub, J. Guzinski, Z. Krzeminski, and H. A. Toliyat, "Advanced control of induction motor based on load angle estimation," IEEE Trans. Ind. Electron., vol. 51, no. 1, pp. 5-14, Feb. 2004.

[2] C. Lascu, I. Boldea, and F. Blaabjerg, "Variable-structure direct torque control-A class of fast and robust controllers for induction machine drives," IEEE Trans. Ind. Electron., vol. 51, no. 4, pp. 785-792, Aug. 2004.

[3] R.-J. Wai and K.-M. Lin, "Robust decoupled control of direct fieldoriented induction motor drive," IEEE Trans. Ind. Electron., vol. 52, no. 3, pp. 837-854, Jun. 2005.

[4] B. H. Bae, B. H. Cho, and S. K. Sul, "Damping control strategy for the vector controlled traction drive," in Proc. 9th Eur. Conf. Power Electron. and Appl., Graz, Austria, 2001.

[5] B. Delemontey, B. Jacquot, C. Iung, B. De Fornel, and J. Bavard, "Stability analysis and stabilisation of an induction motor drive with input filter," in Proc. 6th Eur. Conf. Power Electron. and Appl., Sevilla, Spain, 1995.

[6] K. Pietiläinen, L. Harnefors, A. Petersson, and H.-P. Nee, "DC-link stabilization and voltage sag ride-through of inverter drives," IEEE Trans. Ind. Electron., vol. 53, no. 4, pp. 1261-1268, Aug. 2006.

[7] M. Jänecke, "Steuerverfahren und Steueranordnung für einen Wechselrichter," ABB Patent DE 4110225 A1, 1992.

[8] S. D. Sudhoff, K. A. Corzine, S. F. Glover, H. J. Hegner, and H. N. Robey, Jr., "DC link stabilized field oriented control of electric propulsion systems," IEEE Trans. Energy Convers., vol. 13, no. 1, pp. 27-33, Mar. 1998.

[9] S. F. Glover and S. D. Sudhoff, "An experimentally validated nonlinear stabilizing control for power electronics based power systems," in Proc. SAE Aerosp. Power Syst. Conf., 1998.

[10] A. M. Walczyna, K. Hasse, and R. Czarnecki, "Input filter stability of drives fed from voltage inverters controlled by direct flux and torque control methods," Proc. Inst. Electr. Eng.-Electr. Power Appl., vol. 143, no. 5, pp. 396-402, Sep. 1996.

[11] X. Y. Liu and A. J. Forsyth, "Comparative study of stabilizing controllers for brushless DC motor drive systems," in Proc. IEEE Int. Conf. Elect. Mach. and Drives, May 15-18, 2005.

[12] S. D. Sudhoff, S. F. Glover, P. T. Lamm, D. H. Schmucker, and D. E. Delisle, "Admittance space stability analysis of power electronic systems," IEEE Trans. Aerosp. Electron. Syst., vol. 36, no. 3, pp. 965-973, Jul. 2000.

[13] D. Kim, B. Choi, D. Lee, and J. Sun, "Analysis of input filter interactions in switching power converters," in Proc. 20th Annu. IEEE Appl. Power Electron. Conf. and Expo., Mar. 6-10, 2005, vol. 1, pp. 191-198.

[14] T. Suntio and A. M. Altowati, "Design of EMI filter for stability and performance in switched-mode converters," in Proc. IEEE 35th Аnпи. Power Electron. Spec. Conf., 2004, pp. 3077-3083.
[15] M. Jänecke, R. Kremer, and G. Steuerwald, "Direct self control, a novel method of controlling asynchronous machines in traction applications," in Proc. Rec. EPE-Conf., Aachen, Germany, 1989, pp. 75-80.

[16] D. Maischak, Schnelle Drehmomentregelung im gesamten Drehzahlbereich eines hochausgenutzten Drehfeldantriebs. Düsseldorf, Germany: VDI Verlag, 1995.

[17] H. Mosskull, "Robust Control of an Induction Motor Drive," Ph.D. dissertation, Autom. Control, Dept. Signals, Sensors Syst., Royal Inst. Technol., Stockholm, Sweden, 2006.

[18] H. Mosskull, "Stabilization of an induction motor drive with resonant input filter," in Proc. 11th Eur. Conf. Power Electron. and Appl., Dresden, Germany, 2005.

[19] M. Lörtscher, M. Meyer, A. Schneeberger, and B. Hemmer, Kompatibilitätsuntersuchungen am schweitzerischen 16,7-Hz-Bahnstromnetz, Elekrische Bahnen, 2001. eb 6-7.

[20] H. Mosskull, B. Wahlberg, and J. Galic, "Validation of Stability for an induction machine drive using measurements," in Proc. 13th IFAC Symp. Syst. Identification, 2003.

[21] M. Barenthin, H. Mosskull, H. Hjalmarsson, and B. Wahlberg, "Validation of stability for an induction machine drive using power iterations," in Proc. 16th IFAC World Congr., Prague, Czech Republic, 2005.

[22] P. Terwiesch, T. Keller, and E. Scheiben, "Rail vehicle control system integration testing using digital hardware-in-the-loop simulation," IEEE Trans. Control Syst. Technol., vol. 7, no. 3, pp. 352-362, May 1999.

[23] H. Mosskull, "Stabilization of an induction machine drive," in Proc. 10th Eur. Conf. Power Electron. and Appl., Toulouse, France, 2003.

[24] H. Mosskull, "Some issues on stabilization of an induction machine drive," in Proc. 43rd IEEE Conf. Decision and Control, The Bahamas, 2004, pp. 4441-4446.

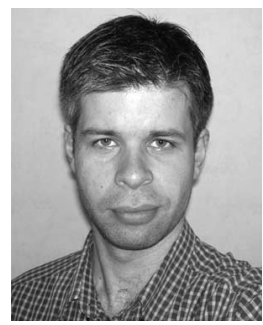

Henrik Mosskull received the M.Sc. degree in electrical engineering from Linköping University, Linköping, Sweden, in 1995, the M.Sc. degree in systems and control engineering from Case Western Reserve University, Cleveland $\mathrm{OH}$, in 1996, and the Ph.D. degree in automatic control from Royal Institute of Technology (KTH), Stockholm, Sweden, in 2006.

In 1996, he joined the Converter Control Group at Bombardier Transportation, Västerås, Sweden. As Converter Control Specialist for medium-power propulsion systems within Bombardier Transportation, he has broad experience with induction motor control through numerous delivery projects. 


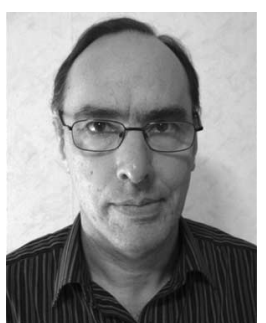

Johann Galić was born in Austria in 1954. He received the M.S. degree in electrical engineering from Chalmers University of Technology, Gothenburg, Sweden, in 1979 .

From 1979 to 1987, he was with ASEA, working with the development of power electronic circuits for industrial and traction applications. From 1987 to 1997, he was with ABB Industrial Systems, working with the control of electrical drives in industrial systems, e.g., rolling mills, paper machines, and cranes. In 1997, he joined the Converter Control Group at Bombardier Transportation, Västerås, Sweden. As Senior Specialist in control, he has broad experience with stability and robustness analysis of propulsion systems.

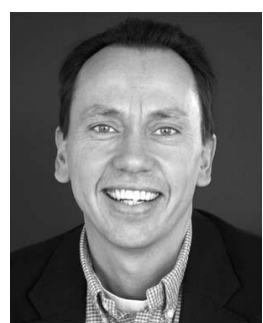

Bo Wahlberg (S'85-M'87-SM'02-F'07) was born in Norrköping, Sweden, in 1959. He received the M.Sc. degree in electrical engineering, the Licentiate degree of Technology, and the Ph.D. degree from Linköping University, Linköping, Sweden, in 1983, 1985 , and 1987 , respectively.

In December 1991, he became a Professor of the Chair of Automatic Control at the Royal Institute of Technology (KTH), Stockholm, Sweden. He was a Fulbright Visiting Professor in the Information Systems Laboratory, Stanford University, Stanford, CA, during August 1997-July 1998, and the Vice President of KTH, during 1999-2001. His research interests include system identification, modeling and control of industrial processes, and signal processing, with applications in communication and autonomous systems. 